\title{
Multi-User Virtual System for Training of the Production and Bottling Process of Soft Drinks
}

\author{
Juan I. Zambrano, David A. Bermeo, Cesar A. Naranjo, and \\ Víctor H. Andaluz \\ Universidad de las Fuerzas Armadas ESPE \\ Sangolquí-Ecuador \\ \{jizambrano5, dabermeo, canaranjo, vhandaluz1\}@espe.edu.ec
}

\begin{abstract}
The project consists of developing a virtual operational training system for the production and bottling process carried out in the soft drinks factory. For the virtualization of the industrial process, mathematical modeling is considered using a transfer function of the first order with downtime and control in relation to the flow plants that make up the substance mixing station in the company, for this, dynamic data and behaviors of the fully automated flow plants within the university are used and not the conventional station existing in the company, since its machines and data are kept confidential In addition, 3D CAD design techniques, electric diagrams of the process and instrumentation (P\&ID) are used, all in order for multiple users to have immersive experiences sensory and can interact with each other within a virtual environment.
\end{abstract}

Keywords -Virtual environment; Production and bottling process; Unity 3D; Multiple users; Training; 4.0 Industry.

\section{INTRODUCTION}

The industrial revolution is a process of economic and social transformation to increase productivity in a company or factory, usually produced by technological progress over time. Just as in the 18 th century it was the steam engine, in the 21 st century it will be robots and virtual environments that will set the tone for a radical transformation, which will change the world as we know it, economists have called it the Fourth Industrial Revolution [1], Industry 4.0 or Fourth Industrial Revolution is a research initiative in Germany to implement the high-tech strategy of integrating Advanced Control Systems with ICT to enable communication between personnel, products and complex systems that make up a Digital Factory [2], considering the technologies that are integrated into the socalled Cyber Physical Systems (CPS Cyber Physical Systems) induce changes in Engineering Systems and Higher Education in Engineering [3] [4].

Taking into account different revisions we can find different elements associated to the development of the industry 4.0 [5]; (i) Big Data and the data cloud: includes algorithms, analysis applications, etc. Big Data analytics manages opportunities for the improvement of future factories, manufacturing processes and enabling the factory to provide new products and services; (ii) Internet of Things: Includes software applications used by one or more businesses to support industrial networks; in addition, intelligent monitoring and control through sensors, smart meters and intelligent mobile devices; (iii) Intelligent manufacturing processes: include dynamic, efficient, automated and real-time process communication for the management and control of a highly dynamic environment enabled by IoT; (iv) Robotics: In industry 4.0 robots acquire skills beyond their predecessors, managing to incorporate new capacities to work without a human supervisor and are able to work to automate and coordinate a series of logistic and production tasks; (v) Virtual Reality (VR): It allows training and information within an Industry, recreating these spaces, environments and interactive virtual situations so that once the training is over, the user knows what he has to do and does it automatically once faced with the real situation.

Currently, the challenge worldwide in fields such as education, management, telecommunications, games, military training or, as in the present work, industrial processes that use VR technology to compete in the market, [6] [7], is to incorporate technologies that are capable of combining the real world of production with the virtual world of information and communication technology; so that, traditional industrial processes are complemented, optimized by the digital world in immersive virtualized environments free of risks and with the ability to simulate the real behavior of the plant or industry, all this thanks to the abundance of information, mobility and connectivity [8].

The incursion of the industry with the VR technology has started from the use of induction videos and virtual tours to train the personnel, without interacting with the industrial processes, until the present time, where it is possible to interact with the processes, to optimize their designs, to provide maintenance, to control the processes and to train the operator [11], giving a focus in the training of the operator, to simulate experiences in the decision making that takes place in the field work [9] [12]. VR allows the user to interact with elements of the virtual world with a realistic approach, in which he has the feeling that he can perform operator simulation under specific conditions in training scenarios [13] [14] [15]. Among the most important advantages of using VR are [9] [10]. (i) Training: The training and education of the personnel is one of the best strategies to control and diminish the index of accidentalness and turns out to be a critical factor for the company; (ii) Induction: It is to give a real approach to new operators so that they can adapt to the technological changes and to the necessity to understand the operation of the surroundings of the company, that allow to assure an effective production of quality, but mainly safe; (iii) 
Telerobotics: consists in the remote handling of robots, but with the exception that the operator sees what the robot is seeing and even has the touch of the machine [13]; (iv) Simulators: used to deal with systems that cannot be handled in the real world. For example, simulations of warlike confrontations, or flight simulators [15] [16]; (v) Risk Analysis: All the activities that are developed in industries, whatever they may be, have dangers and associated risks, in order to control the risks inherent to an activity [9] [10].

Therefore, this work presents the development of a virtual reality system for training in the production and bottling process carried out at the factory and the development of an automatic mixing station with mathematical models and corresponding controllers for the operation in the virtual environment. The components of the factory are designed based on its P\&ID diagram, using 3D design techniques and computer aided design software. The characterization and animation of the objects is implemented in UNITY3D, sounds and interaction are also added to achieve the desired realism and improve the user experience. In addition, for the simulation and control of the syrup and water mixing process in the soft drink factory, the exchange of significant process data between UNITY3D and MATLAB is required, being a fundamental part of this task the respective programming in Matlab, to implement the control algorithm that govern the behavior and evolution of the mixing station.

\section{VIRTUAL ENVIRONMENT}

This article describes the development of the virtualization of the process and bottling plant of soft drinks in a virtual environment with the software Unity 3D through a multi-user collaborative system, where the operators able to interact and have an immersion with the different processes of the plant by means of virtual reality glasses, together with the software tool Matlab that allow the modeling and the control of the industrial process of the plant and techniques of CAD design for construction of the same one.

The virtualization of the process and the bottling environment of soft drinks is developed through CAD design techniques and Unity 3D software to digitize the terrain and the elements of the industrial process, for the virtualization of the environment the facilities of the factory are considered Ambasodas soft drinks, by means of the electrical diagrams and the visualization of the start-up of the company's mixing station, an electronic instrumentation diagram (PI\&D) is generated, with the instruments and devices of the university and data obtained from the dynamic behavior of the Plants involved obtain their mathematical models and control laws that govern the behavior of the mixing station within the virtual environment. MatLab through its instructions allows the exchange and connection of data together with mathematical modeling, in order to simulate the interaction of operators with the virtual environment of the plant through virtual reality glasses that provide an enlarged view of factory and intuitive operator handling with controls and commands, resulting in a tool to train new operators in a safe and risk-free environment. Fig. 1 shows the detailed path that take for the development of this work.

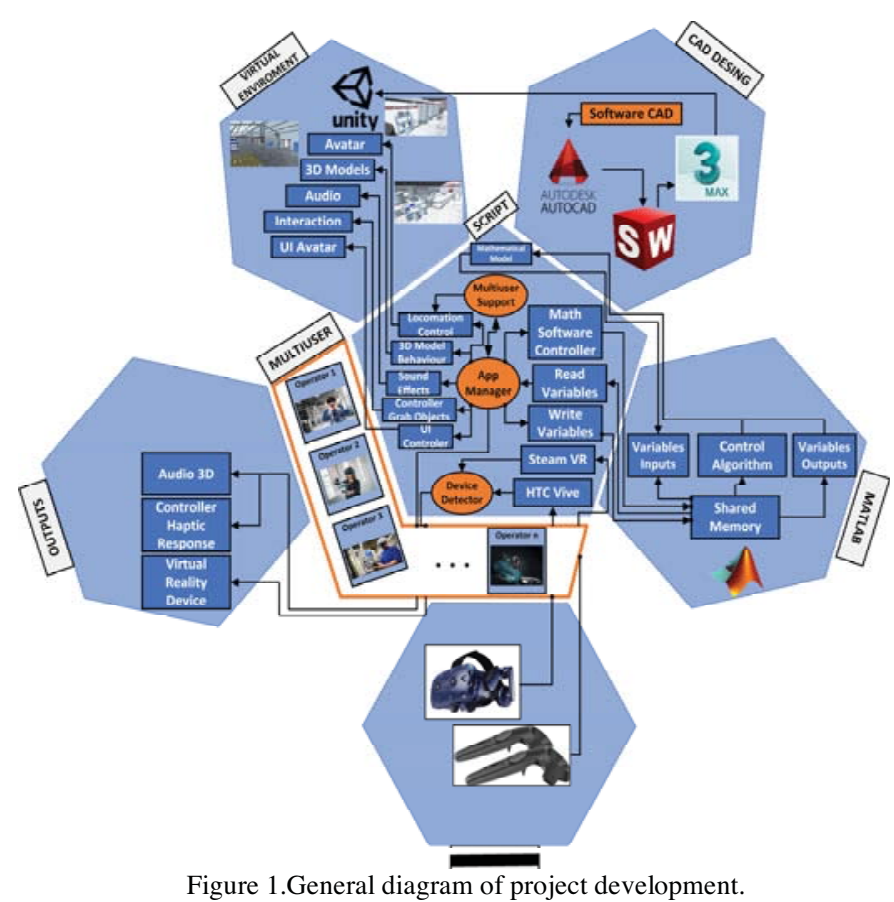

\section{A. MatLab Math Software}

Allows through code lines and control laws to perform the mathematical modeling covering the electrical characteristics in the station of mixing water and syrup, for this the pump that sucks the syrup and the water pump are activated through an HMI, until the storage tank of the mixture detects through its level sensors when it is the right time to empty the tank to the gasification machine.

\section{B. Multi-User}

Multi-user is the stage that relates all the information of input and output from and to users so that operators from different parts of the world can connect to the environment through a communication network and interact with each other, strengthening the collaborative work, see Fig. 2.

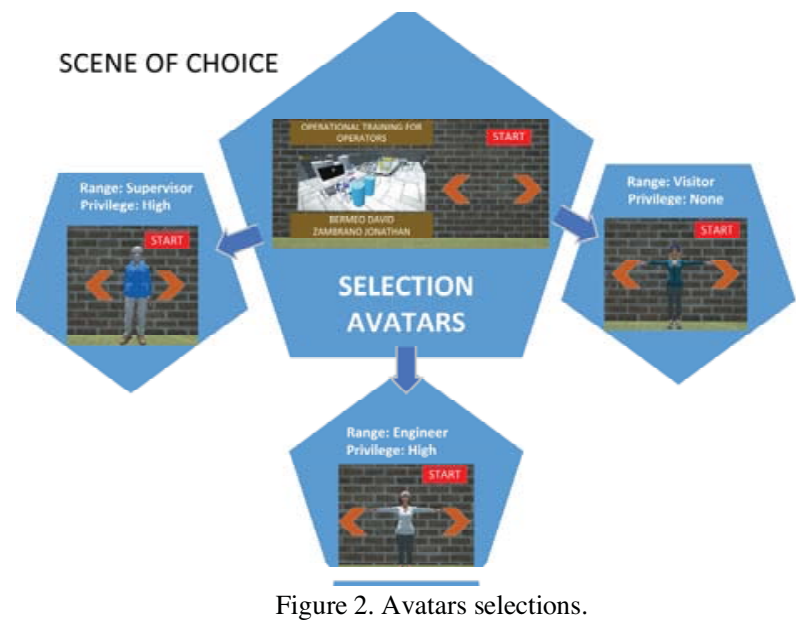

With the use of Unity3D together with Internet the implementation of the multiuser system obtains the information from a database that communicates bidirectionally in a 
synchronized way between the different users, which allow us to access and interact with the process from any location.

The multi-user system was implemented through a Photon Unity network framework for real-time multiplayer games and applications; also, through the scripts in Unity, the creation of a server; which is an instance with authority to coordinate all events. He himself is in charge of encapsulating the data and through calls to remote procedures (RPC) exchange data sent by TCP/IP protocol through Photon SDK to communicate with web services and update the data of tank levels, pumping units, users, he has the database. The system has a login with web services, which were implemented in Google Cloud Platform and with a database created in FireBase. Through scripts, the model of the real dynamic behavior of the tanks and other elements involved are added.

\section{MODELLING AND CONTROL}

In general, any process receives certain changes in inputs and generates certain output responses. It should be clarified that when we talk about inputs and outputs we are not necessarily talking about material flows; on the contrary, the concept is one of information flow, in the sense that inputs are elements that the operator can define or modify, while outputs are determined by the system's responses to certain input conditions.

The operator must decide the actions on the inputs of the process to allow operation in the mixing station specifically in the level control of the main tank; knowing the dynamic response of the system (i.e. how it responds over time) to changes in input conditions is very useful for system management, the process can be better appreciated Many times, when a step signal is introduced at a process input the output signal can approximate a first order function with dead time for both the syrup plant and the water plant.

$$
G_{p}(s)=\frac{K}{\tau_{p} s+1} e^{-\theta_{p} s}
$$

where, $K$ represents the static gain, the relationship between the change in response to the magnitude of the step; $\tau p$ defined the time constant; and $\theta_{p}$ is the time out.

Once the model to which it is possible to approach the mixing station is known, data (tags) are taken of the evolution of the process variables through a set of steps until the mathematical model of the system is obtained.

Next, the modeling of two laboratory flow plants that make up the water and syrup mixing station is carried out by means of the step signal test; in addition the model found is validated by applying the same signal to the entrance. It is worth mentioning that the mathematical models determined in this paper do not represent the dynamic behavior of a real plant of bottling process of soft drinks, but they are useful for re-supplying the factory and implementing the mixing station in the virtual environment.

Figure. 3 shows the dynamic behavior at the output, in the water plant and in the implemented model when a step signal is applied to the input.

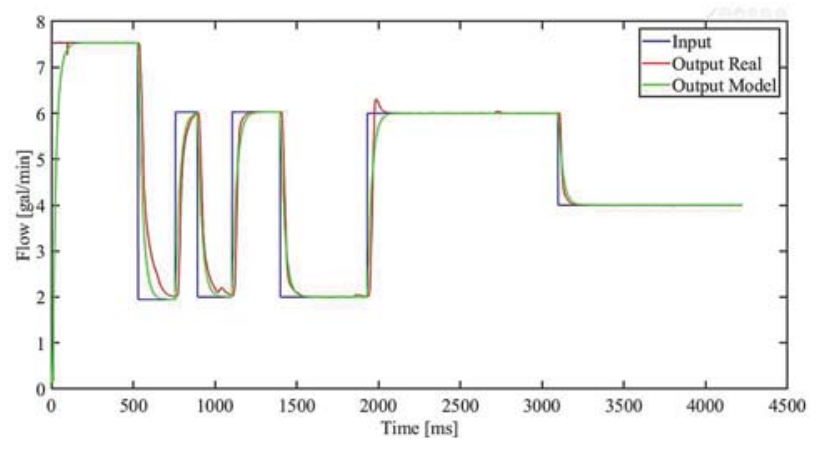

Figure 3. Modeling and Validation of the water plant.

Figure. 4 shows the dynamic behavior at the output, in the syrup plant and in the implemented model when a step signal is applied to the input.

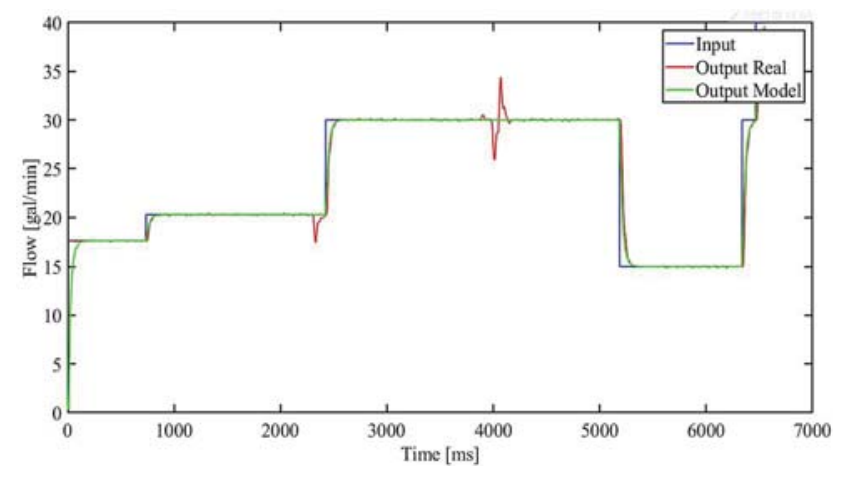

Figure 4. Modeling and Validation of the syrup plant.

The mathematical expressions that characterize the input and output relationships of water and syrup plants are represented by,

$$
\begin{aligned}
& G_{p s}(s)=\frac{1.9211}{1.2172 s+1} e^{-0.7022 s} \\
& G_{p w}(s)=\frac{0.85264}{1.1032 s+1} e^{-1.5492 s}
\end{aligned}
$$

where, $G_{p s}(s)$ represents the transfer function of the syrup plant and $G_{p w}(s)$ is the water plant.

With the objective of solving the dynamics of the water plant as the syrup plant of the virtual environment, (4) and (5) are determined obtaining $G_{p w}(t)$ and $G_{p s}(t)$ in the time domain, respectively.

\section{1) Development of the Controller}

Once the transfer function that govern the plant behavior is obtained, the tuning constants for the PID type digital controller are calculated; the block diagram is shown in Fig. 5, where $r_{1}(t)$ is the water inlet, $r_{2}(t)$ is the syrup inlet, $\left(G_{p w}(t), \mathrm{G}_{p s}(t)\right)$ are the models of plants in the time domain, $\left(y_{1}(t), y_{2}(t)\right)$ are the 
flows of the system output, for this process is considered a constant of relationship defined by the company; which is housed in a Matlab Script and later through shared memories connect the controller process to the $3 \mathrm{D}$ animation of the mixing station. For this device, the PID tuning is taken into account by the Haalman method, which considers the following equations and expressions to calculate the tuning constants.

The PID controller equations show the general equation,

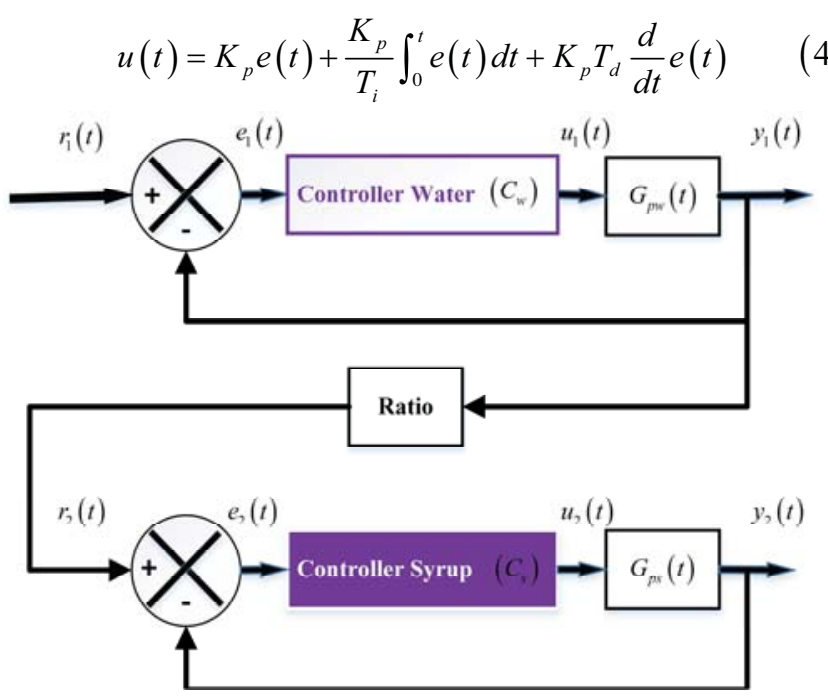

Figure 5. Block diagram of the Ratio controller.

where, $K_{p}$ is the term of the proportional coefficient of the controllers; $e(t)$ is the error signal of the flow processes; $T_{i}$ represents the term of the integral coefficient and $T_{d}$ represents term of the proportional coefficient.

Table I and Table II show three different tuning methods that were applied to the water plant and the syrup plant, where the constants $K_{p}, K_{i}, K_{d}$ are obtained to the plants modeling.

TABLE I. Tuning methods for the water plant.

\begin{tabular}{lccc}
\hline \multicolumn{4}{c}{ Water Plant (a) } \\
\hline & $K_{p}$ & $K_{i}$ & $K_{d}$ \\
Lambda & 0.473 & 0.476 & 0.406 \\
Haalman & 1.097 & 0.645 & 0 \\
Cohen - Coon & 2.508 & 0.455 & 0.359 \\
\hline
\end{tabular}

TABLE II. Tuning methods for the syrup plant.

\begin{tabular}{lccc}
\hline \multicolumn{4}{c}{ Syrup Plant (b) } \\
\hline & $K_{p}$ & $K_{i}$ & $K_{d}$ \\
Lambda & 0.203 & 0.637 & 0.272 \\
Haalman & 0.601 & 0.821 & 0 \\
Cohen - Coon & 1.344 & 0.690 & 0.234 \\
\hline
\end{tabular}

The Fig. 6 shows the evolutions of the different methods applied to water and syrup plants, where we conclude that the Haalman method is the most optimal for modeling.
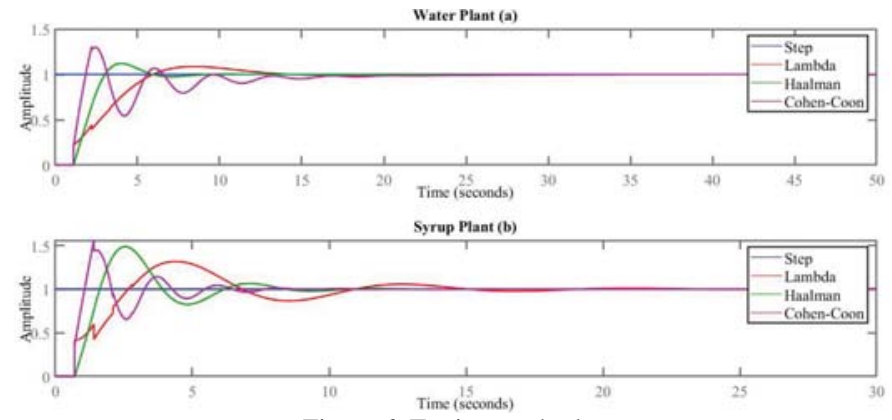

Figure 6. Tuning methods

\section{RESULTS ANALYSIS}

This section presents the performance of a collaborative task in an environment of the soft drink production and bottling process; for the virtualization of the environment the facilities of the factory AMBASODAS soft drinks are considered.

The activity is focused on the interaction of the operator with the mixing station, which allows for a virtualized work experience of the operations performed in the different stages involved in the soft drink production and bottling process. These operations are focused on the mixing of purified water and flavor syrup, contained in the different storage and mixing tanks; specifically, in the main container type tank, as well as the activation of the conveyor belt motors for the filling, capping and labeling of the soft drinks. The different stages of virtualization of the production and bottling process of soft drinks are detailed below.

\section{A. Water Treatment}

At this stage the drinking water is stored in the reception tanks by means of the connected pipes, see Fig. 7, which is sent to impurity filters purifying the water and then stored in a main tank to be taken again to the water and syrup mixing process, see Fig. 8.

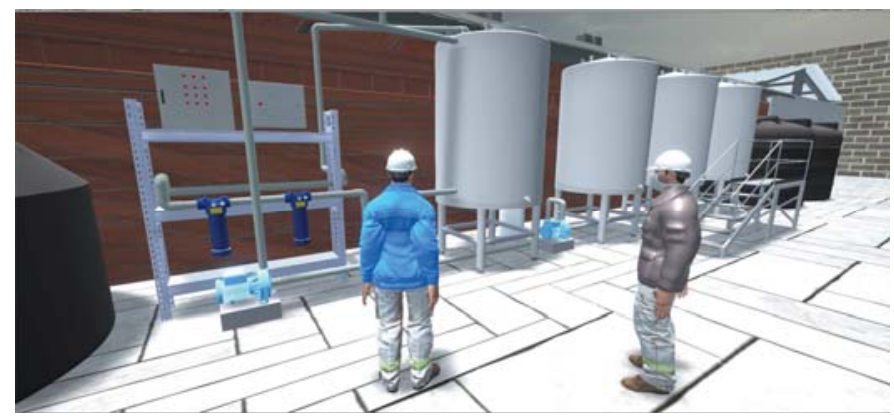

Figure 7. Storage Tanks. 


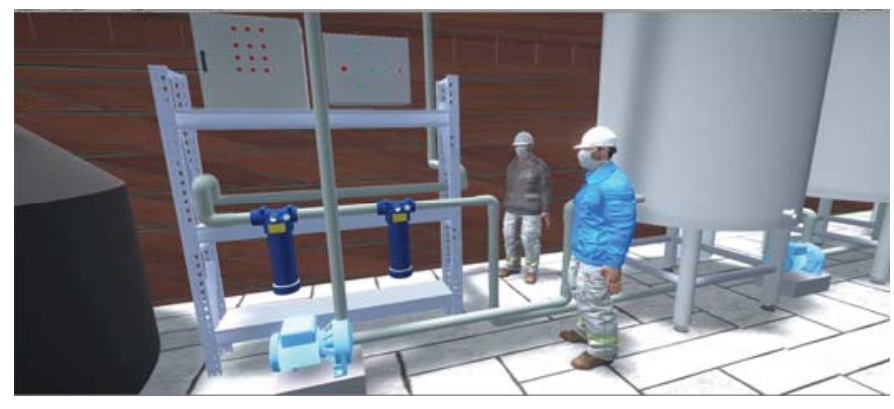

Figure 8. Water Filter.

\section{B. Syrup Reception}

At this stage, the syrup suppliers arrive in trucks and it is stored in the reception tanks inside the plant at room temperature by means of connected pipes, see Fig. 9, where it is ready to be combined in the mixing station with the purified water.

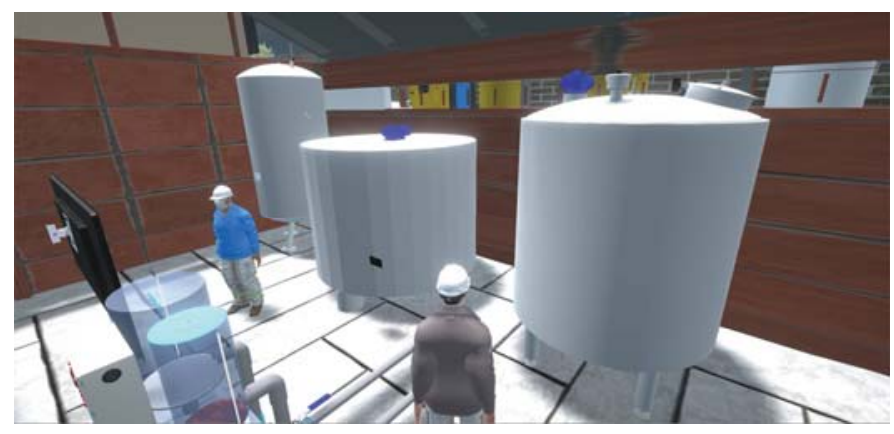

Figure 9. Syrup Reception Tanks.

\section{Syrup-Water Mixture}

At this stage, the purified water and syrup are received in separate tanks, see Fig. 10, with a 10:1 ratio by means of actuators (motors). The process continues when the substances reach the top of their capacity in the mixing station tanks and are released by means of actuators (valves) into another main container to later be sent to the gasification machine and continue with the process.

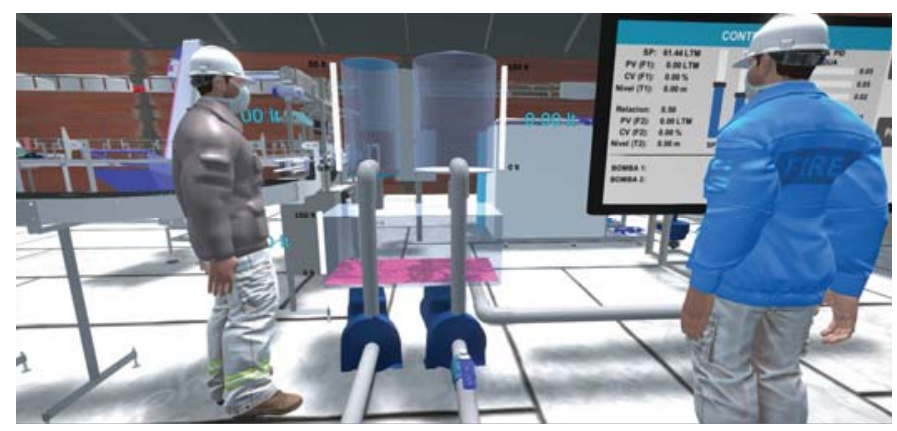

Figure 10. Mixing Station.

\section{Gasifier}

In this stage, it receives the $\mathrm{CO} 2$ that is in the external part of the plant and enters the gasification plant that consists of two tanks, see Fig. 11, the first tank enters the carbon dioxide with the mixture of syrup plus water and through a centrifugal engine it is transported to another tank for the next stage of the process.

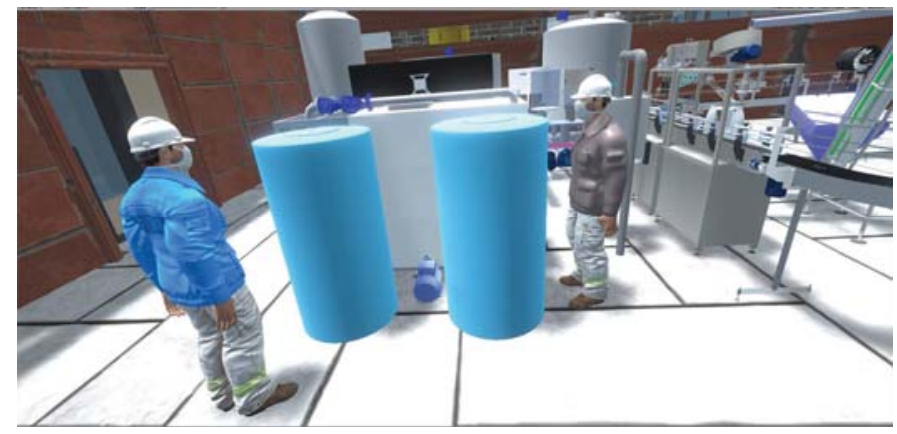

Figure 11. Gasifier Water/Syrup Mixing Tanks.

\section{E. Bottle Filling}

At this stage, the soda is transported to a rotating tank, see Fig. 12, which by means of the machines and the conveyor belt carry out the filling process in the non-returnable containers.

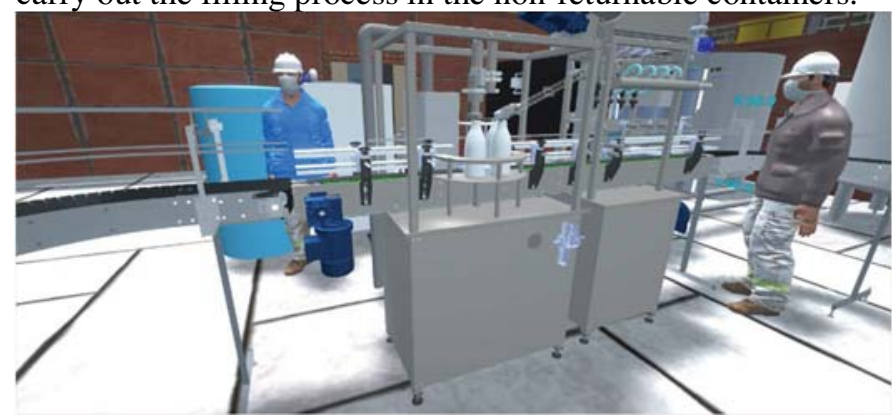

Figure 12. Bottle Filling Stage.

\section{F. Bottle Capping}

At this stage, once the container is full, it is covered, labelled and packed, see Fig. 13, with the factory AMBASODAS brand, ready to be distributed in different parts of the country.

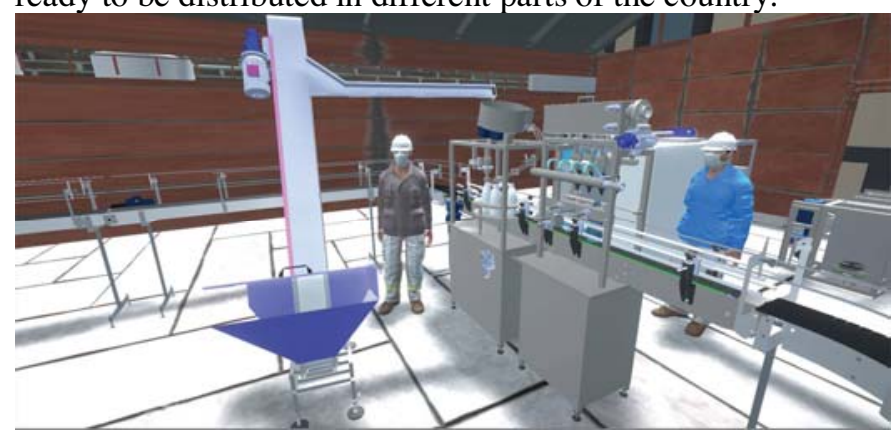

Figure 13. Bottle Capping Stage

To verify the validity of the interface of the multi-user training system application, a focus is given to the effectiveness of the training tasks and the maneuvers in the mixing station, a joint evaluation is applied to 15 users to quantify the results in terms of benefit.

TABLE III. Usability of the virtual environment 
SUS the usability [17] of the training system was evaluated by means of a questionnaire of questions, developing an evaluation in a group of 15 people, among them 7 students, 5 workers of the Ambasodas company and 3 professors of the Electronic Engineering career in the research laboratory, with students who know the topics of virtual reality and process control. In addition, a brief explanation of the hardware to be used for this experience is given, to determine the experience in the virtual training environment [9], as indicated in Table III.

The results obtained determine that (shown in Fig. 14), on average more than 12 of the 15 people evaluated indicate that the virtual application to train operators in the production and bottling process of soft drinks contributes to learning and development of cognitive skills of students and teachers in the area, since it is a novel tool easily accessible as a theoretical and practical support when interacting or performing a task in a virtual reality.

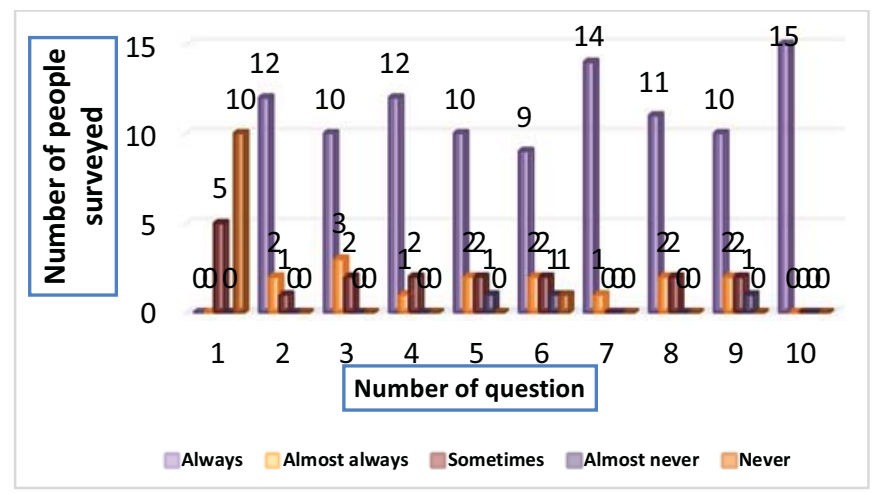

Figure 14. Determination of the level of usability of the application.

\section{CONCLUSIONS}

The creation of the virtual environment immersed in the AMBASODAS factory created for training in the company environment, provides important features such as the ability to make a virtual tour around the facilities and interact in terms of control with the level station that governs the behavior of the plant mixing syrup and water, simulating the response of the process and its respective controller, There are evident advantages with the use of this type of training systems such as the creation of safe situations in critical processes of the mixing station or the familiarization in the stages of production and bottling of soft drinks. In addition, the equation that governs the behavior and evolution through time of the station has been modeled by means of mathematical software techniques to later apply a PID controller to later take those values to Unity and animate the virtual station of mixing syrup and water; The creation of a link between multiple users to interact in the same task is the key to optimal operational training, for all the above mentioned it is established that this system helps in important aspects such as security, process control, increased productivity, operator training, virtual tours, among others.

\section{ACKNOWLEDGMENT}

Q. Questions
Q1. Have you ever used devices HTC VIVE that allow to immersion
Q2. The use of the training module is intuitive?
Q3. Does it result easy the management of devices in the virtual
environment?
Q4. Are you able to perform the operation given by the virtual
assistant?
Q5. Could you identify risk conditions and emergency situations in a
Q6. Are the operating modes in the substance mixing system
Qnderstandable?
Would you recommend the training system as an additional tool to
ine theoretical training that is given in the educational and
Q8. Did the virtual application help you understand and improve skills
in industrial processes?
Q9. Do you have good maneuverability on the dash buttons that control
the operation of the substance mixing station?
Does the incorporation of new technologies (RV) allow the
development of practices with problems of real industrial
processes?

\section{REFERENCES}

[1] López Franco, M. L., Lovato Torres, S. G., \& Abad Peña, G. El impacto de la cuarta revolución industrial en las relaciones sociales y productivas de la industria del plástico Implastic S. A. en Guayaquil-Ecuador: retos y perspectivas. Universidad y Sociedad, 10(5), pp. 153-160, 2018.

[2] Carvajal R, J. "La Cuarta Revolución Industrial o Industria 4.0 y su Impacto en la Educación Superior en Ingeniería en Latinoamérica y el Caribe", Scientia e Technica, Red de Revistas Científicas de América Latina y el Caribe, Universidad Antonio Nariño, Colombia, Vol. 18 (1), 2013.

[3] Reiner Anderl. "Industry 4.0 - Advanced Engineering of Smart Products and Smart Production", Technological Innovations in the Product Development, 19th International Seminar on High Technology (Piracicaba, Brasil October 9th, 2014).

[4] Hightech-Strategy for Germany. (2020). [Online]. Available: http://www.hightech-strategie.de/en/350.php

[5] Davila Gilede, J. , Castillo, D., Sanchez, Pedro. Industria 4.0 y tendencias en la ingeniería industrial, Programa de Industrial en la Universidad Militar Nueva Granada, UMNG. Colombia, 2018.

[6] Nayhua Gamarra,J., Guzman Neyra,R. Uso de Realidad Virtual Inmersiva (RVI) para el entrenamiento de personal en Seguridad, mantenimiento, 2018.

[7] Andaluz V., et. al. Inmerssive Industrial Process Enviroment from a P\&ID Diagram. Internationaal Symposium on Visual Computing, pp. 701-712, 2016.

[8] Pallares R, C. Petrotécnica. (2016, April). Realidad virtual e impresión 3D hidráulica en la industria del petróole y del gas [Online]. Avaliable: http://www.petrotecnia.com.ar/abril16/Sin_Publicidad/Realidad.pdf

[9] Yugcha, E. P., Ubilluz, J. I., \& Andaluz, V. H. Virtual Training for Industrial Process: Pumping System. Augmented Reality, Virtual Reality, and Computer Graphics. doi:10.1007/978-3-030-25999-0_33, pp. 393409, 2019.

[10] Porras, A. P., Solis, C. R., Andaluz, V. H., Sánchez, J. S., \& Naranjo, C. A. Virtual Training System for an Industrial Pasteurization Process. 
Augmented Reality, Virtual Reality, and Computer Graphics. doi:10.1007/978-3-030-25999-0_35, pp. 430-441, 2019.

[11] Zovko, M.E., Dillon, J.: Humanism vs. competency: traditional and contemporary models of education. Educ. Philos. Theor. 50, pp. 554-564, 2017.

[12] Yu, Y., Duan, M., Sun, C.H., Zhong, Z., Liu, H.: A virtual reality simulation for coordination and interaction based on dynamics calculation. Ships Offshore Struct. 12, pp. 873- 884, 2017.

[13] Gonzaga, L., et al.: Immersive virtual fieldwork: advances for the petroleum industry. In: IEEE Computer Society, pp. 561-562, 2018.

[14] Hutton, C., Suma, E.: A realistic walking model for enhancing redirection in virtual reality. In: IEEE Virtual Reality (VR), pp. 183-184, 2016.
[15] Romo J.E., Tipantasi G.R., Andaluz V.H., Sanchez J.S. Virtual Training on Pumping Stations for Drinking Water Supply Systems. In: De Paolis L., Bourdot P. (eds) Augmented Reality, Virtual Reality, and Computer Graphics. AVR 2019. Lecture Notes in Computer Science. Springer, Cham, vol 11614, 2019

[16] Moreno E.F., Pacheco E.E., Andaluz V.H., Mullo Á.S. Multi-user Expert System for Operation and Maintenance in Energized Lines. In: Arai K., Kapoor S., Bhatia R. (eds) Advances in Information and Communication. FICC 2020. Advances in Intelligent Systems and Computing. Springer, Cham, vol 1130, 2020.

[17] J. Sauro y J. R. Lewis, When designing usability questionnaries, does it hurt to be positive, In Proceeding of the SIGCHI Conference on Human Factors in Computing Systems, pp. 2215-2224. 